about small molecule oral direct inhibitor of activated factor $\mathrm{X}$ (FXa) anticoagulant (rivaroxaban and apixaban) gradually carried out. The conclusion has show clear pharmacokinetic and efficacy features. Rivaroxaban showed superior effectiveness in the Clinical study RECORD, making it become the first clinical application anticoagulant and not require anticoagulation monitoring. It is proved that rivaroxaban reduce the mortality from deep vein thrombosis, pulmonary embolism and all-cause by $18.9 \%$. Take off to want: it will not increase the risk of bleeding. Rivaroxaban has been used for preventing vein thrombos in adult elective total hip or total knee replacement. Related trials in the prevention of stroke leading by atrial fibrillation and secondary prevention of acute coronary syndrome (ACS) is in progress. APPRAISE studies show that aspirin or aspirin plus clopidogrel therapy added to $5 \mathrm{mg}$ or $10 \mathrm{mg}$ apixaban may have therapeutic potential in the hope to prevent second heart attack in ACS patients. And the further tests hope the combination based on standard regimen can effectively reduce the cardiovascular events, stroke and mortality in ACS patients. Currently, apixaban clinical trials in prevention of venous thrombosis and prevention of stroke caused by atrial fibrillation are also on going. Current studies tend to show that, compared with placebo, oral factor Xa inhibitor base on standard regimen can give available benefits in ACS patients. But these findings still need further large-scale controlled studies to confirm the statistical significance.

\section{e0462 RELATIONSHIP BETWEEN RED CELL DISTRIBUTION WIDTH AND COMPLICATION RISK IN PATIENTS WITH ACUTE MYOCARDIAL INFARCTION}

doi:10.1136/hrt.2010.208967.462

${ }^{1}$ Kaizu Xu, ${ }^{2}$ Liming Lin, ${ }^{3}$ Jinxiu Lin, ${ }^{1}$ Jiansheng Zheng, ${ }^{1}$ Dingnan Cai. ${ }^{1}$ Medical College of Putian University, Putian; ${ }^{2}$ The Affiliated Hospital, putian University; ${ }^{3}$ Department of Cardiology, The First Affiliated Hospital, Fujian Medical University

Objective To investigate the relationship between red cell distribution width (RDW) level and risk of complication in acute myocardial infarction (AMI) patients; to compare the correlation of RDW with NT-proBNP, cTnI and hs-CRP.

Methods From January 2006 to December 2009, 200 consecutive AMI patients admitted in cardiology department of affiliated hospital of putian university were enrolled. Patients were classified into complication group ( $\mathrm{n}=145$ ) or complication-free group $(\mathrm{n}=55)$ according to the presence or absence of new-onset symptomatic heart failure, arrhythmia or cardiac shock. Patients were divided into quartiles based on RDW value ( $\leq 12.8 \%, 12.9 \%-13.8 \%, 13.9 \%-$ $14.7 \%, \geq 14.8 \%, \mathrm{n}=50$ in each quartile), and OR of incident complication was calculated by using logistic regression. Correlation of RDW with NT-proBNP, cTnI and hs-CRP was compared by spearman rank correlation analysis.

Results RDW levels in complication group was significantly higher than that in Complication-free group $(14.5 \pm 0.97 \%$ vs $12.9 \pm 0.85 \%$, $\mathrm{p}<0.05)$. RDW levels of AMI patients were positively associated with complication risk, after adjustment for estimated glomerular filtration rate, serum ferrum, left ventricular end-diastolic dimension, left ventricular ejection fraction, and plasma NT-proBNP, cTnI and hs-CRP levels, the highest RDW quartile entailed 1.96 times greater risk for complication than the lowest quartile $(95 \% \mathrm{CI}$ 1.34-2.79, P cTnI $>$ hs-CRP ( $r s=0.31,0.29$ and 0.21 respectively, all $\mathrm{p}<0.05)$.

Conclusion Higher RDW is closely associated with increased risk of AMI complication and elevated plasma NT-proBNP and cTnI level.

\section{e0463 RELATIONSHIP BETWEEN RED CELL DISTRIBUTION WIDTH AND COMPLICATION RISK IN PATIENTS WITH ACUTE MYOCARDIAL INFARCTION}

doi:10.1136/hrt.2010.208967.463

${ }^{1}$ Kaizu Xu, ${ }^{2}$ Limin Lin, ${ }^{2}$ Jinxiu Lin, ${ }^{3}$ Jiansheng Zheng, ${ }^{3}$ Dingnan Cai. ${ }^{1}$ Medical College of Putian University; ${ }^{2}$ Department of Cardiology, The First Affiliated Hospital, Fujian Medical University; ${ }^{3}$ Medical College of Putian University Putian

Objective To investigate the relationship between red cell distribution width (RDW) level and risk of complication in acute myocardial infarction (AMI) patients; to compare the correlation of RDW with NT-proBNP, cTnI and hs-CRP.

Methods From January 2006 to December 2009, 200 consecutive AMI patients admitted in cardiology department of affiliated hospital of putian university were enrolled. Patients were classified into complication group $(n=145)$ or complication-free group $(n=55)$ according to the presence or absence of new-onset symptomatic heart failure, arrhythmia or cardiac shock. Patients were divided into quartiles based on RDW value ( $\leq 12.8 \%, 12.9 \%-13.8 \%, 13.9 \%-$ $14.7 \%, \geq 14.8 \%, n=50$ in each quartile), and OR of incident complication was calculated by using logistic regression. Correlation of RDW with NT-proBNP, cTnI and hs-CRP was compared by spearman rank correlation analysis.

Results RDW levels in complication group was significantly higher than that in Complication-free group $(14.5 \pm 0.97 \%$ vs $12.9 \pm 0.85 \%$, $\mathrm{p}<0.05)$. RDW levels of AMI patients were positively associated with complication risk, after adjustment for estimated glomerular filtration rate, serum ferrum, left ventricular end-diastolic dimension, left ventricular ejection fraction, and plasma NT-proBNP, cTnI and hs-CRP levels, the highest RDW quartile entailed 1.96 times greater risk for complication than the lowest quartile (95\% CI 1.34-2.79, P cTnI > hs-CRP ( $\mathrm{rs}=0.31,0.29$ and 0.21 respectively, all $\mathrm{p}<0.05)$.

Conclusion Higher RDW is closely associated with increased risk of AMI complication and elevated plasma NT-proBNP and cTnI level.

\section{e0464 CLINICAL SIGNIFICANCE OF THE CHANGES OF SERUM TNF- $\alpha$, IFN- $\gamma$, MMP-9}

doi:10.1136/hrt.2010.208967.464

Zhang Zhaozhi, Meng Xiaoping. Cardiology The Second Hospital of Jilin University

A variety of inflammatory factors in atherosclerosis (AS) plays a complex role, the combination of two or more of the inflammatory mediators can increase inflammatory mediators in coronary heart disease diagnosis and treatment of value.

Objective To investigate Clinical significance of the changes of serum TNF- $\alpha$, IFN- $\gamma$, MMP-9, oxLDL levels in patients with acute coronary syndrome (ACS).

Method Selected 63 patients who had been done coronary angiography from June 2009 to January 2010 in our hospital. There are 37 males and 26 females, divided into 3 groups: acute myocardial infarction (AMI) group, unstable angina group and the normal control group. According to the results of coronary angiography the patients were divided into 3 groups: acute myocardial infarction group, unstable angina group and the normal control group. There are 27 cases in AMI group, 17 males and 10 females, mean age $(62.5 \pm 11.4)$ years. Selection criteria: According to clinical symptoms, ECG changes, myocardial enzyme increases, coronary angiography, all patients are $\mathrm{Q}$-wave myocardial infarction, and the incidence in the $3 \mathrm{~h} \sim 24 \mathrm{~h}$. There are 20 patients in UAP group, 13 males and 7 females, mean age (64.4 \pm 9.1$)$ years of age. Selection criteria: Overworked deterioration of angina and (or) resting angina, and at least onseted once severe angina in $48 \mathrm{~h}$, electrocardiographic 